\title{
The Immediate Effects of Posterior Pelvic Tilt with Taping on Pelvic Inclination, Gait Function and Balance in Chronic Stroke Patients* Yang-Ting Wu $\cdot$ Yu-Won Choe $\cdot$ Cheng Peng $\cdot$ Myoung-Kwon Kim ${ }^{1 \dagger}$ \\ Dept. of Rehabilitation Sciences, Graduate School, Daegu University \\ ${ }^{1}$ Dept. of Therapy, College of Rehabilitation Sciences, Daegu University
}

Received: April 13, 2017 / Revised: April 13, 2017 / Accepted: May 16, 2017

(C) 2017 J Korean Soc Phys Med

\begin{abstract}
| Abstract |
PURPOSE: The purpose of this study is to identify the immediate effect of posterior pelvic tilt taping on anterior pelvic inclination, gait function, and balance in chronic stroke patients.
\end{abstract}

METHODS: Fourteen chronic stroke subjects were enrolled in this study. Subjects who consented to participate in this cross-over experiment were assigned three interventions: posterior pelvic tilt taping, placebo taping, and no taping, in random order. After tape application, subjects were asked to complete: 1) Anterior pelvic tilt measurement, 2) 10-Meter Walk test, and 3) Limits of stability (LOS) test. To eliminate the learning effect of the tape after tearing off the tape, a 10 minute break was given between posterior pelvic tilt taping intervention and placebo taping intervention.

RESULTS: Significant decreases were observed for the anterior pelvic inclination on both sides after posterior pelvic

\footnotetext{
* This paper is abridgment of thesis for a master's degree of Yang-Ting $\mathrm{Wu}$ (2017).

† Corresponding Author : skybird-98@hanmail.net

This is an Open Access article distributed under the terms of the Creative Commons Attribution Non-Commercial License (http://creativecommons.org/licenses/by-nc/3.0) which permits unrestricted non-commercial use, distribution, and reproduction in any medium, provided the original work is properly cited.
}

tilt taping application compared with placebo taping and no taping application $(p<.05)$. Post hoc test results differed significantly in the 10-meter walk test after intervention $(p<.05)$. However, there were no significant differences in limits of stability test after intervention ( $p>.05)$.

CONCLUSION: Posterior pelvic tilt taping in chronic stroke patients decreases the inappropriate anterior pelvic inclination immediately and improves gait function, but it has little effect on balance.

Key Words: Anterior pelvic tilt, Balance, Gait, Posterior pelvic tilt taping, Stroke

\section{Introduction}

Stroke survivors have difficulty in balance and postural control for standing upright, as they suffer from asymmetric posture, abnormal body imbalance, and insufficient weight transfer (Chung et al., 2013; Yang and Kim, 2017; Ko and Lee, 2017; Song et al., 2010). These problems have a major effect on the patient's gait function (Lee and Lee, 2016; Rojhani-Shirazi et al., 2015). Pelvic motion is an ordinary phenomenon for stroke patients and the importance of this is emphasized in terms of gait economy (Staszkiewicz 
et al., 2012). Pelvic elevation and tilt angle increase occur with stroke hemiplegic gait, and stroke patients usually have poor motor function, resulting in excessive tilt of the pelvis during the stance and swing phase. These changes reduce stability and gait speed (Chen et al., 2003; Dickstein and Abulaffio, 2000; Titianova and Tarkka, 1995). The circumduction gait consists of a combination of hiking and forward rotation of the pelvis (Perry et al., 1992). These evidences highlighted that the importance of sway amplitudes and pelvis stability are crucial in paretic patients, and have influence on circumduction gait pattern of lower limb and locomotor performance, functional ability of upper limb activity, and trunk control of stroke patients (Kerrigan et al., 2000; Bujanda et al., 2003; Verheyden et al., 2011). These research findings emphasize the pelvis's influence on postural control and gait function.

Several therapeutic interventions have been conducted with an aim to correct and control these problems, including selective pelvic exercises (Cabanas-Valdés et al., 2016), body weight-supported treadmill training (Mao et al., 2015), and gait training with rhythmic auditory stimulation (Shin et al., 2015). However, most of the treatment protocols are labor-intensive and require site space, which makes the provision of intensive treatment for all patients difficult. Therefore, an alternative method is introduced in this study to address those problems.

Kinesio taping, which uses elastic adhesive tape with an elasticity rate similar to that of the skin, is being used by physical therapists during rehabilitation programs to treat musculoskeletal pain and functional abnormalities (Briem et al., 2011). Kinesio tape is a thin, highly elastic material that can relieve pain, correct joint malalignment, decrease swelling, increase proprioception, provide support for muscles, and increase or inhibit muscle recruitment (Rojhani-Shirazi et al., 2015; Cepeda et al., 2008). Kinesio taping can improve postural control and gait ability by not only stimulating the proprioceptive sense but also identifying the correct position of the joint (Rojhani-Shirazi et al., 2015; Kim et al., 2014). Baek et al. (2011) reported that motor function on the paretic side significantly improved when hemiplegic stroke patients received Kinesio taping application to the muscles on the paretic side. Kim et al. (2002) also noted that Kinesio taping application in families with hemiplegic stroke patients greatly improved daily living activity, joint range of motion, and hand motions. Other advantages of the Kinesio taping include its low cost and easy application (Kim et al., 2014).

Although Kinesio taping is a part of clinical practice in physical therapy, to the best of our knowledge, no studies have evaluated the application of Kinesio taping to correct anterior pelvic tilt problems on postural control, including balance ability and gait function in chronic stroke patients. For this reason, the objective of this study is to determine whether there are changes on pelvic tilt angle, gait function and balance ability in chronic stroke subjects with anterior tilt pelvis from the data obtained by three different measurements, after applying posterior pelvic tilt taping, placebo taping and no taping intervention respectively.

\section{Methods}

\section{Participants}

This study was conducted on 14 chronic stroke patients from D welfare center, Korea. Prior to the start of study, all subjects understood the content of the study and signed an informed consent form. The study was authorized by the Daegu Research Ethics Committee of Daegu University and the IRB number is 1040621-201611-HR-020-02. This study complied with the ethical standards of the Declaration of Helsinki.

All patients were diagnosed with stroke confirmed with computed tomography or magnetic resonance imaging. The inclusion criteria employed were: 1) chronic stroke involving cerebral regions ( $>6$ months post stroke) with an anterior pelvic inclination $\left(>15^{\circ}\right.$, normal range $11 \pm 4^{\circ}$ ) (Hagins et 
al., 1998; Levine and Whittle, 1996); 2) Brunnstrom stage of motor recovery for the affected lower limb range of $3-5 ; 3)$ ambulatory but with residual gait deficit as defined as a visual gait abnormality assessed by a licensed physical therapist (including those who use a cane or walker); 4) ability to understand and follow simple verbal instructions; 5) independent gait ability to walk at least $15 \mathrm{~m}$ without assistance; 6) no disability in visual, auditory, and vestibular organs; 7) no history of orthopedic diseases, such as contracture, fracture, or arthritis in lower limbs and 8) a Mini-Mental State Examination score greater than 24/30 (Folstein et al., 1975). Exclusion criteria were as follows: 1) neurological problems other than stroke that would interfere with gait and balance control; 2) pain, limited motion, or weakness in the non-paretic lower extremity that affected performance of daily activities (by self-report), and 3) allergy to the Kinesio tape or having a skin disease. The necessary sample size, 14 study subjects, was calculated based on the data from a pilot study that had a minimum power of $80 \%$ with an alpha level of $95 \%$.
This study was conducted as a randomized controlled trial with cross-over design. Each subject was assigned to posterior pelvic tilt taping, placebo taping and no taping according to a randomization plan created with a generator from Google (http://www.randomization.com). This randomization was performed by a third party unaware of study content. To eliminate the effect of the previous intervention, a 10 minute-break was given between posterior pelvic tilt taping and placebo taping intervention. Subjects were required to perform specific experiments to collect three different measurements (Fig. 1).

\section{Intervention}

The posterior pelvic tilt taping was applied by a single physical therapist fully trained in the Kinesio taping method. The elasticity of the kinesiology tape (BB TAPE, WETAPE Inc., Seoul, Korea) is approximately a $70 \%$ to $80 \%$ stretch above the original length. According to the stretch guidelines provided by Kase et al. (2003), the tension for facilitating muscle function is $25 \%$ to $50 \%$ of the avail-

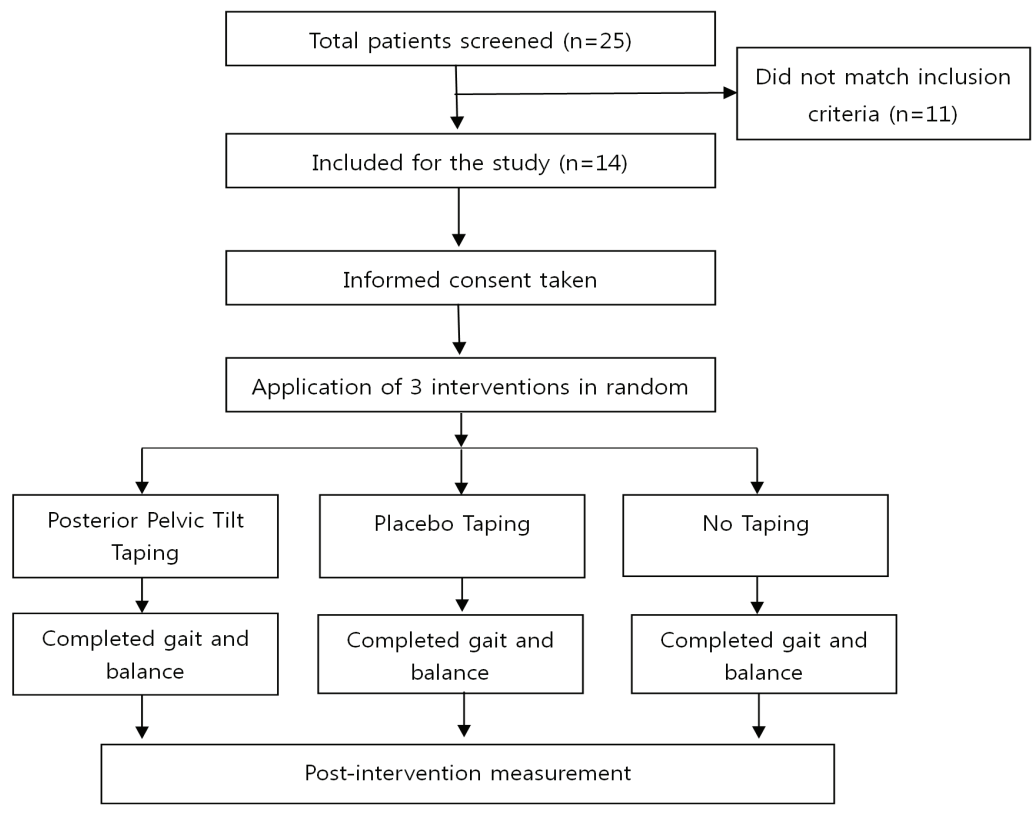

Fig. 1. Study flowchart

PPTT: Posterior Pelvic Tilt Taping; PT: Placebo Taping; NT: No Taping 
able tension, and $50 \%$ to $75 \%$ of the available tension is used for mechanical correction. I-type strips with approximately $50 \%$ of the available tension were placed over both the rectus abdominis (RA) and external oblique (EO) muscles, which are involved in posterior pelvic tilting, for facilitating muscle function (Kendall et al., 2005). I-type strips with approximately $75 \%$ of the available tension were applied from the anterior superior iliac spine (ASIS) to the posterior superior iliac spine (PSIS) to provide a mechanical correction effect (i.e., posterior tilt to the pelvis bilaterally).

The published posterior pelvic tilt taping methodology (Lee et al., 2014) calls for the first strip of tape to be applied over the EO muscle, originating in the inguinal region and terminating on the spinous process of the twelfth thoracic vertebra. The second strip of tape originating at the ASIS was pulled over the PSIS. When applying these two tapes, the participant was in a side-lying position, and the pelvis was maintained in the posterior pelvic tilt posture so that the participant would not experience pain while using the tape. The third tape strip, which was applied over the RA, originated near the pubic symphysis and terminated on the xiphoid process and fifth to seventh costal cartilages, while the participant was in the hook-lying position. The direction of the tape application is shown by the arrow. Furthermore, participants were instructed to remove the tape immediately if they experienced itching of the skin underneath it.

The placebo taping was applied by the same physical therapist with one I-type strip originating near the position above the ASIS, wrapping around the iliac crest and terminating above the PSIS while the participant was in a side-lying position. The tape was applied without tension.

Participants were naive about the Kinesio taping method, and it was explained that they were receiving two types of therapeutic taping applications. For reducing the margin of error as much as possible, all taping was applied by Physician 1 who was trained in the Kinesio taping method. The assessments were completed by Physician 2 who was blinded to this study. Both of the physical therapists attended the relevant training on assessment procedures, one day before starting the experiment.

\section{Outcome measures}

\section{1) Pelvic Tilt measurement}

A palpation meter (PALM, Performance Attainment Associates, St. Paul, MN, USA), which consists of an inclinometer and two caliper arms, was employed to measure the anterior inclination of the pelvic. The inclinometer is a semicircular arc with one degree gradations that range from $0^{\circ}$ to $30^{\circ}$ on either side of the midline. During the measurement, the participants wore non-restrictive clothing, removed their shoes, and spread their feet (approximately 10 12 cm). They stood in an upright position, leaning the anterior aspect of the thighs against a stabilizing table (Gnat et al., 2009). The investigator palpated the prominence of the ipsilateral ASIS and PSIS and marked them with a black pen. The anterior pelvic tilt was measured by placement of the caliper tips of the PALM in contact with the ipsilateral ASIS and PSIS (Lee et al., 2011). The PALM is a valid, reliable, and cost-effective clinical measurement instrument used to calculate the height discrepancy between landmarks (da Costa et al., 2010; Petrone et al., 2003). Several studies have used PALM to measure static innominate rotation of the ipsilateral ASIS and PSIS (Krawiec et al., 2003; Preece et al., 2008). The intra-test reliability of the PALM is 0.90 , and the inter-test reliability is 0.85 . To minimize error, the change in pelvic inclination between ASIS and PSIS was measured by the same individual. An anterior pelvic tilt angle is shown as a positive $(+)$ value, and a posterior pelvic tilt angle as a negative (-) value.

\section{2) 10-Meter Walk test}

For the ten-meter walk test, $10 \mathrm{~m}$ was measured on the floor using a tape measure. The tape was attached to 
mark the start and end points. The walking course consisted of $14 \mathrm{~m}$ in a hallway: a 2-m warm-up, $10 \mathrm{~m}$ used for the speed measurement, and $2 \mathrm{~m}$ for slowing down to a stop. Instructions were provided to the participant to "walk at a comfortable pace." Participants were provided up to maximum assist by one person for balance and stability (but not for paretic-limb advancement). Participants used the assistive device (e.g., cane, walker) or orthotic device (e.g., ankle-foot orthosis) that they used "most often" (if any) at each time point (Tilson et al., 2010). Three trials were conducted in succession, with a brief seated or standing rest as needed by the participant between trials. For the $10-\mathrm{m}$ walking test, test-retest reliability is reported at .95 and inter-rater reliability at .90 (Mehrholz et al., 2007).

\section{3) BioRescue}

A BioRescue (RM Ingenierie, Rodez, France) was used to assess the balance ability by measuring the moving distance and area of the center of pressure (Baek and Kim, 2014). BioRescue includes a platform $\left(610 \times 580 \times 10 \mathrm{~mm}^{3}\right)$ equipped with 1,600 pressure sensors that measure vertical pressure fluctuations in both feet. The force movements of the foot reflect fluctuations in the foot center of pressure. The patient steps onto the BioRescue equipment, putting their heels together so that their second toes were situated on the diagonal line of the foot pad, and maintaining the anterior parts of their feet at 30 degrees. Patients stood in their most comfortable posture on the measurement equipment, with both arms held relaxed beside their trunk. Limits of stability test, which has good test-retest reliability (ICC= .78-91) for patients with stroke (Liston and Brouwer, 1996; Chien et al., 2007), measured dynamic postural stability using a BioRescue system. Subjects were asked to move their body's center of gravity as far as possible in the following eight directions (left, right, forward, backward and toward the midpoints of each direction) as indicated by arrows. LOS test was measured for 10 seconds using their dominant leg in the left, right, forward, and backward directions. Three trails were performed and the average value was calculated for analysis.

\section{Statistical Analysis}

All statistical analyses were performed using SPSS (IBM Corp. Released 2012. IBM SPSS Statistics for Windows, Version 21.0. Armonk, NY: IBM Corp.). The dependent variables were pelvic inclination, balance and gait ability. Repeated one-way ANOVA was used to determine whether there were changes in anterior pelvic inclination, balance and gait function with posterior pelvic tilt taping, placebo taping and no taping intervention in each subject. Null hypotheses of no difference were rejected if p-values were less than .05 .

\section{Results}

A summary of clinical and demographic features of the study subjects $(\mathrm{n}=14)$ is provided in Table 1 . Subject characteristics compared with the outcome measurements that were conducted after taping are shown in Table 2, Table 3, and Table 4. The anterior pelvic tilt angle on both sides significantly decreased $(p<.05)$ after the posterior pelvic tilt taping application compared with placebo taping and no taping application, and similarly, the time subjects spent on the 10-meter walk test differed significantly $(\mathrm{p}<.05)$ (Fig. 2). The treatment did not produce a difference in the LOS test (Fig. 2).

Table 1. General subject characteristics $(n=14)$

\begin{tabular}{cc}
\hline Variable & Mean \pm SD \\
\hline Age (year) & $64.29 \pm 7.39$ \\
Height $(\mathrm{cm})$ & $163.36 \pm 10.40$ \\
Weight $(\mathrm{kg})$ & $61.86 \pm 14.98$ \\
Sex (male/female) & $7 / 7$ \\
Duration (month) & $94.50 \pm 22.88$ \\
\hline
\end{tabular}

SD: standard deviation 
Table 2. Comparison of the anterior pelvic tilt angle by treatment $(n=14)$

\begin{tabular}{lccccc}
\hline & \multicolumn{3}{c}{ Mean \pm SD } & \multirow{2}{*}{ F } & p \\
\cline { 2 - 4 } & NT & PT & PPTT & & \\
\hline Lt. ASIS-PSIS Tilt Angle & $17.64 \pm 2.13$ & $16.07 \pm 2.13$ & $7.36 \pm 1.91$ & 312.901 & $<.05$ \\
Rt. ASIS-PSIS Tilt Angle & $15.43 \pm 2.17$ & $14.14 \pm 2.38$ & $7.29 \pm 2.33$ & 86.370 & $<.05$ \\
\hline
\end{tabular}

NT: No Taping; PT: Placebo Taping; PPTT: Posterior Pelvic Tilt Taping

Table 3. Comparison of the 10-meter walk test by treatment $(n=14)$

\begin{tabular}{|c|c|c|c|c|c|}
\hline & \multicolumn{3}{|c|}{ Mean $\pm \mathrm{SD}$} & \multirow{2}{*}{$\mathrm{F}$} & \multirow{2}{*}{$\mathrm{p}$} \\
\hline & NT & PT & PPTT & & \\
\hline 10 -meter walk test (s) & $13.06 \pm 3.84$ & $12.78 \pm 4.16$ & $11.95 \pm 3.64$ & 11.843 & $<.05$ \\
\hline
\end{tabular}

NT: No Taping; PT: Placebo Taping; PPTT: Posterior Pelvic Tilt Taping

Table 4. Comparison of the Limits of Stability test by treatment $(n=14)$

\begin{tabular}{|c|c|c|c|c|c|}
\hline & \multicolumn{3}{|c|}{ Mean $\pm \mathrm{SD}$} & \multirow{2}{*}{$\mathrm{F}$} & \multirow{2}{*}{$\mathrm{p}$} \\
\hline & NT & PT & PPTT & & \\
\hline Total area $\left(\mathrm{mm}^{2}\right)$ & $2294.93 \pm 2312.23$ & $2504.14 \pm 2349.85$ & $2683.50 \pm 2245.93$ & .691 & NS \\
\hline
\end{tabular}

NT: No Taping; PT: Placebo Taping; PPTT: Posterior Pelvic Tilt Taping; NS: not significant
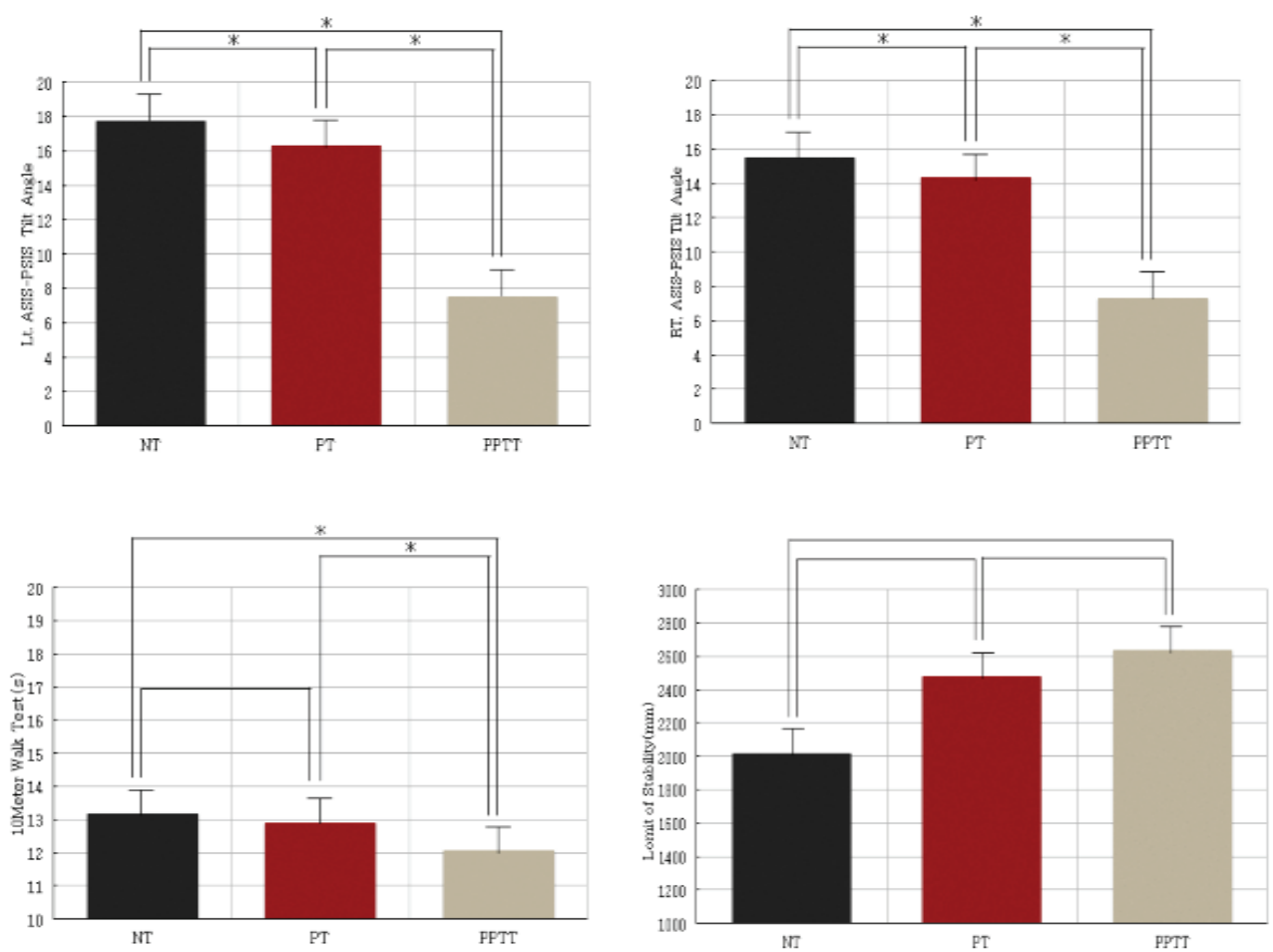

Fig. 2. Comparison of the anterior pelvic tilt angle (Lt/Rt), 10-meter walk test and limit of stability test among three different taping methods $\left({ }^{*} p<.05\right)$. 


\section{Discussion}

Compensatory anterior pelvic tilt is often observed in stroke patients accompany with poor motor function especially during the walking phase (Dickstein and Abulaffio, 2000; Titianova and Tarkka, 1995). Given this, this preliminary study hypothesized that posterior pelvic tilt taping application to chronic stroke patients will produce changes in pelvic inclination, gait function and balance.

In this study, the anterior pelvic tilt on both sides dropped immediately after posterior pelvic tilt taping application compared with initial scores and scores obtained after placebo taping application. The mechanical correction technique with Kinesio tape may be used to assist the positioning of muscle, fascia tissue, or joints in the desired position (Kase et al., 2003). Consequently, in the present study, the tension produced by an I-type strip with approximately $75 \%$ of the available tension applied from ASIS to the PSIS, which acts as a preload in the end-of-motion positions (Kase et al., 2003), might provide a mechanical correction effect, resulting in the pelvis tilted posteriorly.

Comparable to the method in our study, Lee and Yoo (2012) reported that pelvic taping applied to a female amateur swimmer who had an anteriorly tilted pelvis produced positive effects on pelvic inclination and sacral horizontal angle. In that study, Kinesio tape was applied to the RA muscle to reduce both lumber lordosis and the bilateral activation of the lateral fibers of the EO muscle which flexed the vertebral column. All of those changes caused a posterior pelvic tilt. Kase et al. (2003) proposed that the stimulation that Kinesio tape with an elasticity of $30 \sim 40 \%$ above the original length applied around the pelvis can activate a nerve impulse, causing local depolarization that triggers nerve impulses to travel along the afferent fibers to the central nervous system. In other words, the elasticity of Kinesio tape may stimulate cutaneous mechanoreceptors (Murray and Husk, 2001), strengthen weakened muscles, and normalize muscle functions (Kase et al., 1996). Moreover, Kinesio tape may affect the muscle and myofascia functions by increasing lymph circulation at the point of application (Kase et al., 1996). These findings might explain the slight changes in pelvic inclination after placebo taping application compared with no taping application. In summary, considering the points above, the RA and EO muscle, to which Kinesio tape was applied in this study, might produce a posterior pelvic tilt by increasing bioelectric activity. These results suggest that posterior pelvic tilt taping intervention could favorably decrease the excessive anterior pelvic inclination.

With gait ability, the total time that subjects spent in 10-meter walk test decreased according to the changes observed after the application of posterior pelvic tilt taping, placebo taping and no taping. Trueblood et al. (1989) pointed out that the major gait deviations of the pelvis and hip observed in the hemiplegic subjects were excessive pelvic anterior tilt, which occurred throughout the gait cycle and these changes persisted throughout the single-limb support period. In our experiment, the subjects had posterior pelvic tilt taping applied, which adjusted their pelvises to a more normal position. The subjects with posterior pelvic tilt taping spent less time in walking 10 meters compared with control applications. Wade et al. (1986) reported that the 10-meter walking velocity test for hemiplegic patients is a simple, objective measurement to evaluate functional recovery. This finding supported our research; thus it can be interpreted that the decrease of anterior pelvic tilt after posterior pelvic tilt taping application accelerates walking speed and enhances gait ability.

Forward and backward pelvic tilt frequently affect walking in stroke patients (Kong et al., 2015), and abnormal pelvic tilt is associated with gait ability. Jeong and Yoon (2006) proposed that pelvic displacement triggers difficulty in maintaining balance and normal gait, and restricts social activity. Titianova et al. (1995) described the pelvic elevation of the affected side during swing with hemiplegic patients, and demonstrated an association between the circumduction gait and pelvic deviation amplitude. Mao 
et al. (2015) indicated that the decrease of the anterior inclination in pelvis would help the patients regain their normal gait pattern. Those results indicate the importance of improving the gait ability during walking by maintaining or adjusting the neutral posture of the pelvis.

The improvements in gait function in our study observed after applying the posterior pelvic tilt taping, are largely in line with previous studies. Therefore, we conclude that the hypothesis that posterior pelvic tilt taping application affects the patients' gait ability by adjusting the position of pelvis to some extent is proven.

However, no significant differences in balance ability were found from the LOS test. Park found that pelvis intervention does not affect balance ability and stable gait (Park et al., 2015). Another study investigated the walking and balance of stroke patients after Kinesio taping application, with the result for balance ability had no significant statistical difference (Kim et al., 2014). A balance study proposed that maintenance of balance relies on the harmonious integration and coordination of multiple body systems including the vestibular, visual, auditory, sensory, and autonomic systems (Matsumura and Ambrose, 2006). The information from these different end organs is integrated in the central nervous system, and an appropriate response is formulated, causing the musculoskeletal system to directly perform the appropriate head, eye, trunk, and limb movements to maintain posture (Konrad et al., 1999). Therefore, it seems to be difficult to improve the balance ability if the posture is only adjusted by controlling the position of pelvis. Nevertheless, there were several studies in where the balance ability improved immediately after taping intervention (Lee et al., 2016).

A number of limitations warrant attention when interpreting our results. Subjects with a wide range of number of years post-stroke (61-138 months) and two types of stroke are included in our study. More detailed and clinically applicable information could be supplied by narrowing this range or including more participants in different stages suffered from stroke, and classifying them by type of stroke. Apart from this, the geographical limitation should be considered. Specifically, the study participants were approached via a rehabilitation center, therefore the subjects may not represent all chronic stroke patients. Furthermore, the data obtained in this study represent only the immediate effect of posterior pelvic tilt taping in stroke patients. Therefore, further studies with a longer treatment period and a follow-up after the treatment are required to investigate the associations among balance, gait and other functional activities with pelvic position control.

\section{Conclusion}

Our study shows that posterior pelvic tilt taping produces significant therapeutic effects on the inappropriate anterior pelvic inclination in chronic stroke patients. Moreover, it improves gait function, but has little effect on balance.

\section{References}

Baek IH, Kim BJ. The effects of horse riding simulation training on stroke patients' balance ability and abdominal muscle thickness changes. J Phys Ther Sci. 2014; 26(8):1293-6.

Baek YH, Seo JC, Lee JD. The effect of taping therapy on the activity of daily living of poststroke-hemiplegic patients: a clinical study. The acupuncture. 2011;18.

Briem K, Eythörsdöttir H, Magnúsdóttir RG, et al. Effects of kinesio tape compared with nonelastic sports tape and the untaped ankle during a sudden inversion perturbation in male athletes. J Orthop Sports Phys Ther. 2011;41(5):328-35.

Bujanda ED, Nadeau S, Bourbonnais D, et al. Associations between lower limb impairments, locomotor capacities and kinematic variables in the frontal plane during 
walking in adults with chronic stroke. J Rehabil Med. 2003;35(6):259-64.

Cabanas-Valdés R, Bagur-Calafat $\mathrm{C}$, Girabent-Farrés $\mathrm{M}$, et al. The effect of additional core stability exercises on improving dynamic sitting balance and trunk control for subacute stroke patients: a randomized controlled trial. Clin Rehabil. 2016;30(10):1024-33.

Cepeda JP, Fishweicher A, Gleeson M, et al. Does Kinesio Taping of the abdominal muscles improve the supine-to-sit transition in children with hypotonia. Retrieved on April. 2008;12:2011.

Chen CL, Chen HC, Tang SF, et al. Gait performance with compensatory adaptations in stroke patients with different degrees of motor recovery. Am J Phys Med Rehabil. 2003;82(12):925-35.

Chien $\mathrm{CW}$, Hu MH, Tang PF, et al. A comparison of psychometric properties of the smart balance master system and the postural assessment scale for stroke in people who have had mild stroke. Arch Phys Med Rehabil. 2007;88:374-80.

Chung EJ, Kim JH, Lee BH. The effects of core stabilization exercise on dynamic balance and gait function in stroke patients. J Phys Ther Sci. 2013;25(7):803-6.

da Costa BR, Armijo-Olivo S, Gadotti I, et al. Reliability of scapular positioning measurement procedure using the Palpation Meter (PALM). Physiotherapy. 2010;96(1):59-67.

Dickstein R, Abulaffio N. Postural sway of the affected and nonaffected pelvis and leg in stance of hemiparetic patients. Arch Phys Med Rehabil. 2000;81(3):364-7.

Folstein MF, Folstein SE, McHugh PR. "Mini-mental state": a practical method for grading the cognitive state of patients for the clinician. J Psychiatr Res. 1975;12(3):189-98.

Gnat R, Saulicz E, Biały M, et al. Does pelvic asymmetry always mean pathology? Analysis of mechanical factors leading to the asymmetry. J Hum Kinet.
2009;21:23-32.

Hagins M, Brown M, Cook C, et al. Intratester and intertester reliability of the palpation meter (PALM) in measuring pelvic position. J Man Manip Ther. 1998;6(3):130-6.

Jeong HS, Yoon JG. The Effects of Pelvic Tilt Exercise on Balance of Hemiplegic Patients. Phys Ther Korea. 2006;13(3):41-8.

Kase K, Tatsuyuki H, Tomoko O. Development of Kinesio tape. Kinesio taping perfect manual. Kinesio Taping Association. 1996;6:117-8.

Kase K, Wallis J, Kase T. Clinical therapeutic applications of the Kinesio taping method. Kinesio Taping Association. 2003.

Kendall FP, McCreary EK, Provance PG, et al. Muscles: testing and function, with posture and pain $\left(5^{\text {th }} \mathrm{ed}\right)$. Baltimore. Lippincott Williams \& Wilkins. 2005.

Kerrigan DC, Frates EP, Rogan S, et al. Hip hiking and circumduction: quantitative definitions. Am J Phys Med Rehabil. 2000;79(3):247-52.

Kim KS, Seo HM, Lee HD. Effect of taping method on ADL, range of motion, hand function \& quality of life in post-stroke Patients for 5 weeks. Korean J Rehabil Nurs. 2002;5(1):7-17.

Kim WI, Choi YK, Lee JH, et al. The effect of muscle facilitation using kinesio taping on walking and balance of stroke patients. J Phys Ther Sci. 2014; 26(11):1831-4.

Ko YJ, Lee HS. The Effect of Weight-shift Training with Hula Hoop on Weight Shift Change and Gait in Stroke Patients: A Cross - Sectional Pilot Study. J Korean Soc Phys Med. 2017;12(1):9-14.

Kong SW, Jeong YW, Kim JY. Correlation between balance and gait according to pelvic displacement in stroke patients. J Phys Ther Sci. 2015;27(7):2171-4.

Konrad HR, Girardi M, Helfert R. Balance and aging. The Laryngoscope. 1999;109(9):1454-60.

Krawiec CJ, Denegar CR, Hertel J, et al. Static innominate asymmetry and leg length discrepancy in asymp- 
tomatic collegiate athletes. Man Ther. 2003;8(4): 207-13.

Lee HM, Lee JA. The Effects of Dual-task Action Observation Physical Training on the Walking Ability and Activities of Daily Living in Chronic Stroke Patients. J Korean Soc Phys Med. 2016;11(2):83-91.

Lee JH, Yoo WG, Gak HB. The immediate effect of anterior pelvic tilt taping on pelvic inclination. J Phys Ther Sci. 2011;23(2):201-3.

Lee JH, Yoo WG, Kim MH, et al. Effect of posterior pelvic tilt taping in women with sacroiliac joint pain during active straight leg raising who habitually wore high-heeled shoes: a preliminary study. J Manipulative Physiol Ther. 2014;37(4):260-8.

Lee JH, Yoo WG. Application of posterior pelvic tilt taping for the treatment of chronic low back pain with sacroiliac joint dysfunction and increased sacral horizontal angle. Phys Ther Sport. 2012;13(4):279-85.

Lee YJ, Kim JY, Kim SY, et al. The effects of trunk kinesio taping on balance ability and gait function in stroke patients. J Phys Ther Sci. 2016;28(8):2385-8.

Levine D, Whittle MW. The effects of pelvic movement on lumbar lordosis in the standing position. J Orthop Sports Phys Ther. 1996;24(3):130-5.

Liston RA, Brouwer BJ: Reliability and validity of measures obtained from stroke patients using the Balance Master. Arch Phys Med Rehabil. 1996;77:425-30.

Mao Y, Chen P, Li L, et al. Changes of pelvis control with subacute stroke: A comparison of body-weightsupport treadmill training coupled virtual reality system and over-ground training. Technol Health Care. 2015;23(s2):S355-64.

Matsumura BA, Ambrose AF. Balance in the elderly. Clin Geriatr Med. 2006;22(2):395-412.

Mehrholz J, Wagner K, Rutte K, et al. Predictive validity and responsiveness of the functional ambulation category in hemiparetic patients after stroke. Arch Phys Med Rehabil. 2007;88(10):1314-9.
Murray H, Husk L. Effect of kinesio taping on proprioception in the ankle. J Orthop Sports Phys Ther. 2001; 31(1):A-37.

Park BS, Kim JH, Kim MY, et al. Effect of a muscle strengthening exercise program for pelvic control on gait function of stroke patients. J Phys Ther Sci. 2015;27(3):641-4.

Perry J, Davids JR. Gait analysis: normal and pathological function. J Pediatr Orthop. 1992;12(6):815.

Petrone MR, Guinn J, Reddin A, et al. The accuracy of the palpation meter (PALM) for measuring pelvic crest height difference and leg length discrepancy. J Orthop Sports Phys Ther. 2003;33(6):319-25.

Preece SJ, Willan P, Nester CJ, et al. Variation in pelvic morphology may prevent the identification of anterior pelvic tilt. J Man Manip Ther. 2008;16(2):113-7.

Rojhani-Shirazi Z, Amirian S, Meftahi N. Effects of ankle kinesio taping on postural control in stroke patients. J Stroke Cerebrovasc Dis. 2015;24(11):2565-71.

Shin YK, Chong HJ, Kim SJ, et al. Effect of rhythmic auditory stimulation on hemiplegic gait patterns. Yonsei Med J. 2015;56(6):1703-13.

Staszkiewicz RO, CHWAŁA W, Forczek WA, et al. Three-dimensional analysis of the pelvic and hip mobility during gait on a treadmill and on the ground. Acta Bioeng Biomech. 2012;1:12.

Song $\mathrm{CH}$, Lee GC, Yoo JH, et al. The Relation Between Postural Sway and Asymmetric Weight-bearing for Fall Prevention in Patients with Stroke. J Korean Soc Phys Med. 2010;5(1):81-8.

Tilson JK, Sullivan KJ, Cen SY, et al. Meaningful gait speed improvement during the first 60 days poststroke: minimal clinically important difference. Phys Ther. 2010;90(2):196

Titianova EB, Tarkka IM. Asymmetry in walking performance and postural sway in patients with chronic unilateral cerebral infarction. J Rehabil Res Dev. 1995;32(3): 236.

Trueblood PR, Walker JM, Perry J, et al. Pelvic exercise and 
gait in hemiplegia. Phys Ther. 1989;69(1):18-26. Verheyden G, Van Duijnhoven HJ, Burnett M, et al. Kinematic analysis of head, trunk, and pelvis movement when people early after stroke reach sideways. Neurorehabil Neural Repair. 2011;25(7):656-63.

Wade DT, Wood VA, Heller A, et al. Walking after stroke.
Measurement and recovery over the first 3 months. 1986;19(1):25-30.

Yang YP, Kim SJ. The Effect of Action Observation with Observation Type on Limits of Stability and Dynamic Gait Ability in Stroke Patients. J Korean Soc Phys Med. 2017;12(1):67-74. 
